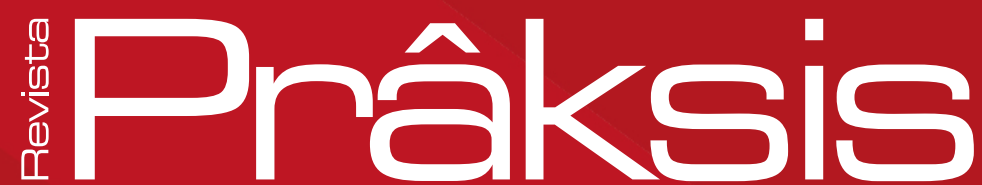

\title{
INNOVATIONS RADICALE ET INCRÉMENTALE : UNE RÉFLEXION SOUS LA PERSPECTIVE DE LA THÉORIE DU CHAOS
}

INOVAÇÃO RADICAL E INCREMENTAL: UMA REFLEXÃO NA PERSPECTIVA DA TEORIA DO CAOS

\section{Eric Charles Henri Dorion}

Doutor em Business administration pela Université de Sherbrooke (2003).

Professor na Universidade de Caxias do Sul (Caxias do Sul/Brasil).

E-mail: echdorion@gmail.com.

\section{Paula Patricia Ganzer}

Doutora em Administração pela Pontifícia

Universidade Católica do Rio Grande do Sul (Porto Alegre/Brasil).

Coordenadora do Curso de Administração na Faculdade CNEC Farroupilha.

E-mail: ganzerpaula@gmail.com.

\section{Uiliam Hahn Biegelmever}

Doutor em Administração pela Pontifícia

Universidade Católica do Rio Grande do Sul (Porto Alegre/Brasil).

E-mail: uilicara@gmail.com.

\section{Adrieli Alves Pereira Radaelli}

Doutoranda em Administracão pela Universidade

de Caxias do Sul (Caxias do Sul/Brasil).

E-mail: adrieli.radaelli@gmail.com.
Joel Tshibamba Mukendi

Mestrando em Administração pela

Universidade de Caxias do Sul (Caxias do Sul/Brasil).

E-mail: tshibambajoel@yahoo.fr.

\section{Cassiane Chais}

Doutoranda em Administração pela Universidade de Caxias do Sul (Caxias do Sul/Brasil).

E-mail: cassichais@gmail.com.

\section{Pelayo Munhoz Olea}

Doutor em Administração e Direção de

Empresas pela Universitat Politècnica de Catalunya (Espanha). Professor na Universidade de Caxias do Sul (Caxias do Sul/Brasil).

E-mail: pelayo.olea@gmail.com.

Maria Emilia Camargo

Doutora em Engenharia de Produção

pela Universidade Federal de Santa Catarina.

E-mail: kamargo@terra.com.br. 


\section{RÉSUMÉ}

Les typologies d'innovation analysent la relation entre le degré de nouveauté de l'innovation et ses impacts sur l'organisation. Deux types reconnus sont l'innovation radicale et incrémentale. L'objectif de cette réflexion théorique est d'analyser deux concepts sous la persepective de la théorie du chaos. Ce travail a pour objectif identifier comment l'innovation radicale et incrémentale sont discutées et comprises dans la littérature scientifique. Dans la perspective exploratoire, une recherche bibliométrique a été réalisée pour soutenir les propositions initiales. Les résultats indiquent l'existence de la standardisation dans l'approche de typologies que traitent des innovations radicales e incrémentales.

Mots-clés: Principe du chaos. Stratégie du principe du chaos. Innovation radicale. Innovation incrémentale.

\section{RESUMO}

As tipologias de inovação têm se preocupado em analisar a relação entre o grau de novidade da inovação e seu impacto na organização. Uma das tipologias mais difundidas foi a inovação radical e incremental. 0 objetivo do ensaio teórico foi analisar estes dois níveis de inovação sob a perspectiva da teoria do caos. Tal proposta visou identificar como os níveis de inovação radical e incremental são abordados na literatura. Por meio de uma pesquisa de abordagem qualitativa, objetivo exploratório e procedimento técnico de revisão de literatura, o método de pesquisa utilizado se baseou em uma pesquisa bibliométrica para fundamentar as proposições apresentadas, cujos resultados apontam a existência de uma padronização quanto a abordagem das tipologias de inovação radical e incremental.

Palavras-chave: Princípio do caos. Estratégia do principio do Caos. Inovação radical. Inovação incremental. 


\section{INTRODUCTION}

Les organisations cherchent la différenciation dans la perspective de l'innovation en vue de se positionner dans le marché concurrentiel actuel (NELSON, 1993; COUTINHO, FERRAZ, 1995; CHRISTENSEN, 2002). Selon le Manuel d'Oslo (OCDE, 2010), I'innovation consiste en une amélioration significative de produit (bien ou service), du processus, de la commercialisation des produits ou de la méthode organisationnelle.

Pour l'apparition des innovations, la dépendance ne se doit pas à une personne ou à une organisation, mais plutôt à un groupe articulé, de sorte qu'une ou plusieurs innovations proviennent du système d'innovation, à savoir, l'ensemble d'agents, les ressources, les politiques et les technologies, bien articulés à cet objectif commun (FREEMAN, 1995; NELSON, 1993).

Dans la définition de l'analyse et de la gestion des changements dans les organisations, la théorie du principe du chaos a été utilisé comme un moyen de réflexion, que stipule que les organisations oscillent sur le principe du chaos, dans lequel il y a comme un constant défi de trouver l'équilibre entre la complète flexibilité et l'absolue inflexibilité des facteurs organisationnels. Le principe du chaos contient les caractéristiques, qui sont: l'incident de comportements compliqués, l'existence des règles de base, la nécessité de travailler à maintenir cet équilibre, l'apparition de surprises et l'apparition d'erreurs, dues au glissement du principe (BROWN; EISENHARDT, 2004).

En considérant les typologies de l'innovation, cette réflexion théorique identifie les différentes formes d'innovation, telleq qu'elles sont classifiées dans la littérature à partir de la perspective de la théorie du chaos. L'article a pour objectif final de clarifier la signification des typologies radicales et incrémentales de l'innovation, ainsi que de proposer une standardisation de typologies dans la littérature.

\section{RÉFÉRENCE THÉORIQUE}

\subsection{INNOVATIONS INCRÉMENTALE ET RADICALE}

Dosi (1982) se réfère les innovations incrémentales au progrès technologique, bien que l'innovation radicale implique la destruction de la compétence qui peut mener à un changement de paradigme. D'une part, une innovation incrémentale peut impliquer un changement dans la forme d'organiser les ressources additionnelles d'une organisation, la création et la mise en valeur de nouvelles versions d'un produit. D'autre part, une innovation radicale peut inclure une nouvelle application d'une combinaison de technologies en nouvelles opportunités de marché (TUSHMAN; NADLER, 1986). Les innovations de Hendersone et Clark (1990), qui sont divisées en quatre catégories en fonction de leur degré d'intégration au système, discute 
comment les innovations incrémentales affectent celles déjà existantes. Les définitions de l'innovation sont plusieurs fois décrites en termes d'étape et de nouveauté (GARCIA; CALANTONE, 2002).

Un cadre théorique encore pertinent aujourd'hui est celui de Christensen (2006), qui traite des termes de soutien et d'innovation perturbatrice, dans laquelle une innovation soutenue améliore la valeur pour le client, lui offrant une maximisation de la performance du produit. Outre les concepts des innovations incrémentale et radicale, qui produisent une innovation " modulaire " et, qui impliquent une réforme des organisations, se sa propre structure a la natures de ses produits, on retrouve aussi l'innovation architecturale à laquelle les composantes sont combinées de façon à repenser ses flux et ses connexions.

En plus de réaliser des améliorations mineures en permanence, les entreprises compétitives arrivent aussi à s'adapter à des innovations radicales ou discontinue, caractérisé par un degré élevé de nouvelle connaissance et d'incertitude (LEIFER et al., 2000; HENDERSON, CLARK, 1990; DEWAR, DUTTON, 1986). La capacité d'une entreprise de chercher et d'explorer afin de s'adapter au fil du temps au changement de conditions se définissent comme des organisations ambidextres (O'REILLY; TUSHMAN, 2004). L'acte d'équilibre des organisations garantit l'efficacité à court terme et l'efficacité à long terme (MARCH, 1991).

Le Manuel d'Oslo (2010) classifie l'innovation incrémentale par des changements " insignifiants " dans les produits et processus, ou encore qui n'impliquent pas un degré suffisant de nouveauté. Stamm (2003) détaille les différences entre l'innovation incrémentale et radicale conformément à différentes perspectives. L'innovation incrémentale se produità court terme. Elle suit une trajectoire de développement de six à 24 mois. Elle se réalise d'étape en étape, depuis la conception de l'objet à sa commercialisation. Elle est le fruit de générations d'idées et de la reconnaissance d'opportunités possédant le flux continu d'améliorations incrémentale, de processus formels et établis.

Les innovations radicales sont abordées dans la perspective des capacités existantes de l'innovation, se renouvelant et se substituant entièrement par des nouvelles compétences, tandis que les activités de benchmarking sur la concurrence, ainsi que la coopération avec les fournisseurs se focalisent sur les innovations incrémentales (ATUAHENE-GIMA, 2005). La coopération entre les universités et entre concurrents sont fondamentales dans la génération de nouveau produits sur le marché, en améliorant la performance des entreprises innovantes (BELDERBOS; CARREE; LOKSHIN, 2004). La génération d'innovation dans les relations de la chaîne d'approvisionnement, autant incrémentale que radicale, est une conséquence d'interactions entre acheteurs et vendeurs. Dès lors les innovations radicale et incrémentale sont classifiées comme un type d'innovation (ROY; SIVAKUMAR; WILKINSON, 2004; ANDRIOPOULOS; LEWIS, 2009). 
Les concepts d'innovations radicale et incrémentale sont également abordés dans la perspective de la création de connaissances et sont classifiés en degré de nouveauté en recherche et de développement (POPADIUK; CHOO, 2006). La codification de la connaissance est bénéfique pour le développement d'innovations incrémentales seulement, spécifiquement lorsque les entreprises intègrent des systèmes de protection juridique dans leurs pratiques de codification afin de maintenir leurs innovations exclusives (GARCÍA-MUIÑA; PELECHANO-BARAHONA; NAVAS-LÓPEZ, 2009).

L'approche descriptive, le comportement du système et le type d'innovation, ainsi que les processus de développement de nouveaux produits individuels peuvent être capables de changer ou d'alterner en différents comportements, notamment, du linéaire au chaotique, afin de produire des innovations. Conformément aux attentes du marché, les types d'innovation se positionnent d'une réalité incrémentale jusqu'à des situations disruptives, ou radicals (MCCARTHY; TSINOPOULOS; ALLEN; ROSE-ANDERSSEN, 2006; COCCIA, 2012).

Dans tous les domaines, la competition-coopération peut améliorer l'appropriation de la valeur en développant les marchés actuels, en facilitant le développement de nouveaux marchés et de nouveaux modèles commerciaux (BOUNCKEN et al., 2018).

\subsection{LA THÉORIE DU PRINCIPE DU CHAOS}

Brown et Eisenhardt (2004) constituent la base de ce travail, en prenant comme concept central la compétition dans le principe du chaos, le noyau central de la théorie qui se définit en trois bases:

a. le principe du chaos: est décrit comme un état naturel entre l'ordre et le chaos, un grand accord entre la structure et la surprise, c'est-à-dire, ce principe est une " espèce de stage " semi-structuré. À ce stade les stratégies et les respectives organisations sont suffisamment rigides pour que des changements puissent se produire, mais pas si rigides pour les en empêcher.

b. le principe du temps: le changement demande la considération des expériences du passé, la concentration dans l'exécution du présent et tout de même la pensée à l'avenir.

c. le contrôle du temps: le changement arrive à travers le passage du temps, et non pas par la survenance des évènements ponctuels.

"La limite du chaos est l'endroit où il y a autant d'emphase sur la concurrence et l'adaptation du modèle existant que sur la création et le développement de nouveaux modèles dans un contexte d'équilibre entre la maintenance, le développement et l'innovation" (MARIETTO et al., 2006, p. 10). Selon Stacey (1995), 
les organisations sont des systèmes complexes e adaptatifs. C'est seulement de cette manière qu'elles arrivent à survivre, grandir et se développer dans la limite entre l'ordre et le désordre, c'est-à-dire, en fonctionnant au maximum de leur capacité, dans un état de d'équilibre constant.

Selon Brown et Eisenhardt (2004), les racines intellectuelles du principe du chaos comptent sur deux présuppositions. La première, c'est que le marché est en constante évolution. II n'y a pas d'espace pour l'équilibre statique, mais plutôt pour la vision selon laquelle les concurrents viennent et vont. Les marchés augmentent et diminuent, se divisent, se heurtent et voire finissent. Les racines intelectuelles de la stratégie, selon le principe du chaos, se basent dans la recherche d'un comportement adaptatif complexe, via le changement évolutif et les origines de la vitesse, pour cela qu'être pionnier devient important. La deuxième présupposition est que les entreprises sont composées de nombreuses parties ou agents et, lorsque ces parties se connectent dans les principes du chaos et du temps, elles forment des systèmes adaptatifs complexes.

Sur la base de ces attributs, Brown et Eisenhardt (2004) cherchent à illustrer la fragilité du principe du chaos, exprimé dans la représentation d'une figure en format d'hyperbole, où le point le plus élevé de celui-ci signifie la " scène " où il y a le principe entre le chaos et la structure, dont la délocalisation accentuée pour n'importe quel côtés, peut forcer une organisation à l'excès de structure ou à la totale absence de celle-ci. Ce type de mouvement peut représenter, selon les auteurs, les pièges du chaos et de la bureaucratie, comme il s'observe dans la Figure 1. 
Figure 1 - Le principe de l'improvisation

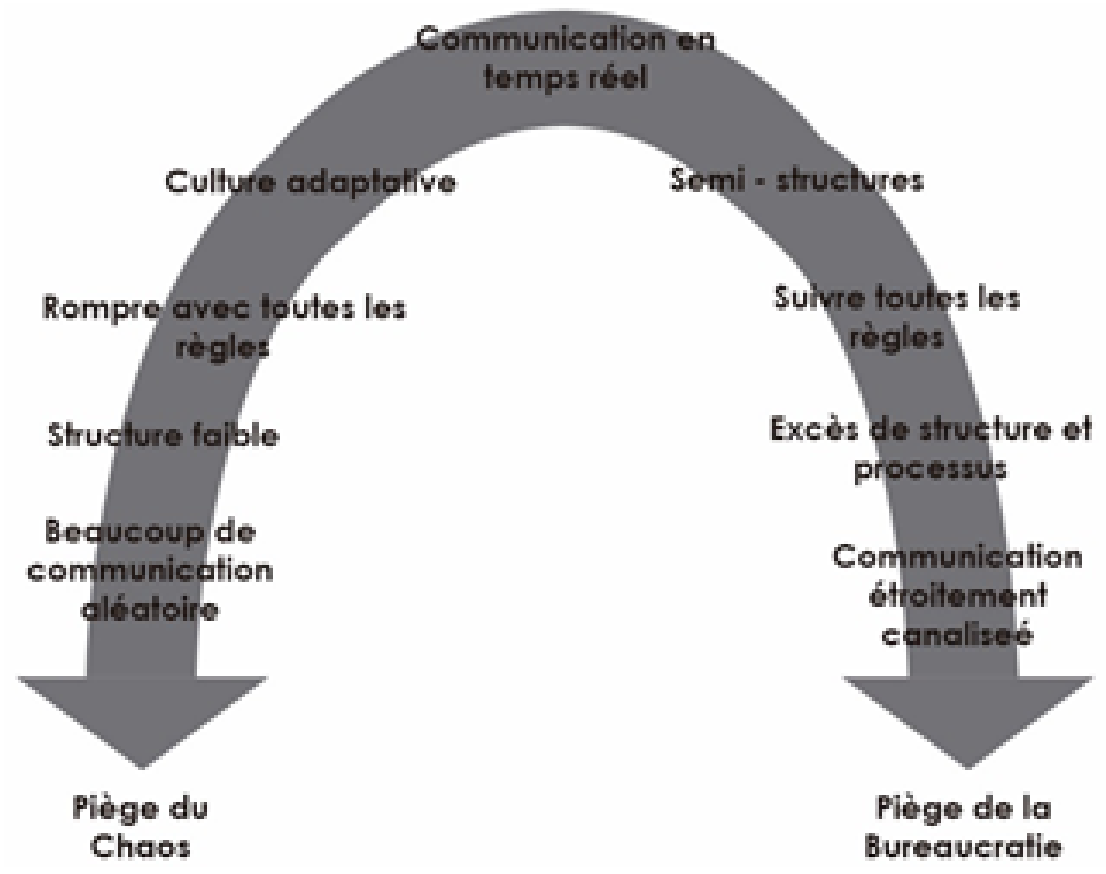

Source: adaptée de Brown et Eisenhardt (2004, p. 46)

En ce sens, Brown et Eisenhardt (2004) expliquent que les positionnements fortement polarisés, d'un côté à l'autre, peuvent exposer une organisation aux pièges du chaos ou de la bureaucratie, comme suit:

a. le piège du chaos: le côté gauche de la figure 1 représente le piège du chaos. L'organisation peut être attirée par une impulsion de fomenter la créativité et l'innovation en vue de l'obtention de changements.

b. le piège de la bureaucratie: le côté droit de la figure 1 représente le piège dans la bureaucratie. Sur ce point, l'organisation est caractérisée par la recherche du processus, la structuration des routines et des contrôles. Un autre concept adapté par Brown et Eisenhardt (2004), dans un contexte stratégique, est celui de l'équilibre dissipatif. Selon ce concept, le système n'est pas techniquement en équilibre mais plutôt en déséquilibre organisé. Selon Brown et Eisenhardt (2004), ce qu'il y a de plus important dans l'approche de la concurrence dans le principe du chaos est que la stratégie est quelque chose résultant de la capacité de l'entreprise à changer constamment et, la capacité 
d'ouvrir de l'espace pour l'émergence d'une direction stratégique. La direction stratégique se développe de manière semi-cohérente et différemment de ce qu'on appelle traditionnellement stratégie. Brown et Eisenhardt (2004) la distinguent par:

c. I'imprévisible: la compétition vient du principe du chaos et implique un élément de surprise.

d. I'incontrôlé: il ne s'agit pasde recourir à la planification de précision ni au commandement des principaux cadres supérieurs de l'entreprise. II y a simplement des choses qui se passent dans les industries, des choses qui changent rapidement, impliquant que chaque action soit orchestrée par un groupe particulier à un moment particulier.

e. I'inefficace: la concurrence dans le principe du chaos n'est pas nécessairement efficace à court terme, elle est une stratégie qui présuppose réussir dans des mauvais marchés, commettre des erreurs, faire demi-tour et entrer dans les bons marchés.

f. le proactif: se concurrencer dans le principe du chaos n'a rien à voir avec attendre passivement I'interruption occasionnelle, ni attendre que d'autres entreprises aient avant de prendre l'initiative.

g. en continu: il s'agit d'imprimer avec le temps, un rythme aux actions, et de ne pas agir d'une manière désarticulée et non systématique.

h. le diversifié: dans le principe du chaos pour concurrencer, il faut assumer les différentes initiatives d'échelles et des risques variables. Plutôt, elle consiste à créer une stratégie robuste et diversifiée, qu'inclut plusieurs initiatives. « Certaines seront brillantes, la plupart seront bonnes et certaines seront mauvaises « (BROWN; EISENHARDT, 2004, p. 22).

Brown et Eisenhardt (2004) défendent le maintien de l'état du principe du chaos, en affirmant que les organisations qui arrivent à transiger avec ce principe obtiennent des performances remarquables au-dessus de la moyenne de l'industrie et changent la stratégie de la même manière que changent les conditions du milieu. Marietto et al (2006) corroborent avec cette vision, expliquant qu'en agissant à la limite du chaos, les organisations peuvent être des meilleurs concurrents et des meilleurs développeurs. En ce sens, il ne suffit pas pour une organisation d'avoir simplement une stratégie; elle doit aussi avoir des alternatives stratégiques.

\subsection{BIBLIOMÉTRIE}

La bibliométrie consiste en l'application des techniques statistiques et mathématiques pour décrire les aspects de la littérature et des autres moyens de communication, c'est-à-dire, l'analyse quantitative de l'information (ARAÚJO, 2006). En bibliométrie, il existe trois lois fondamentales: la loi de Lotka, la 
loi de Bradford et la loi de Zipf. La loi de Lotka postule que le nombre d'auteurs publiant $X$ articles est inversement proportionnel au carré des articles publiés : $n X=n 1 / X^{2}$, où $X=1,2,3 \ldots X$ maximum. Cette loi établit qu'une large proportion de la littérature scientifique est issu d'une poignée d'auteurs.

La deuxième loi bibliométrique se base sur des ensembles de revues scientifiques, afin de découvrir l'extension dans laquelle les éléments d'un sujet scientifique spécifique apparaissaient dans des périodiques destinées pour d'autres sujets, par l'étude de la distribution des articles, en termes de variables de proximité ou d'éloignement. Bradford a réalisé une série d'études qui culminent en 1934 avec la formulation de la loi de dispersion. L'auteur a remarqué que, dans une collection des périodiques sur la géophysique, il existe toujours un plus petit noyau des périodiques généralement liés de près au sujet et un plus grand noyau des périodiques étroitement lié au sujet, où le nombre des périodiques dans chaque domaine de recherche augmente mais où la productivité diminue.

La troisième loi bibliométrique est la loi de Zipf, formulée en 1949, qui décrit la relation entre les mots dans un texte donné suffisamment grand et l'ordre de série de ces mots. La proposition démontre que, lorsque sont listés des mots qui se produisent dans un texte en ordre décroissant de fréquence, la position d'un mot dans la liste, multipliée par leur fréquence est égale à une constante. L'équation pour cette relation se présente comme suit: $r X f=k$, où « $r$ « est la position du mot, « $f$ « est la fréquence et « $\mathrm{k}$ « représente la constante (ARAÚJO, 2006).

Cependant, le domaine le plus important de la bibliométrie est l'analyse des citations, c'est-à-dire, de I'ensemble d'une ou plusieurs références bibliographiques qui, comprises dans une publication, montrent des liens entre les individus, les institutions et les domaines de recherche, car ils révèlent la relation d'une publication avec une autre. La bibliométrie tout au long de son évolution a fini par s'approprier le concept de l'analyse des auteurs, en établissant une corrélation entre les indices absolus de citation à la quantité de travaux cités. L'utilisation du facteur d'impact pour l'évaluation de la production scientifique continue à être pertinente et peut-être parfois liée à d'autres facteurs tels que l'obsolescence et l'âge des références (MEADOWS, 1999; STREHL, 2005).

Dans cette recherche, le nombre de citations des articles a été la condition requise pour la sélection des articles à être analysés. Lima et al., (2009) évaluent la qualité de citation et attribuent l'importance du point de vue en fonction de la quantité de fois qu'un auteur est cité, mais pas nécessairement la qualité de l'ouvrage. C'est-à-dire, la publication d'une citation dans un périodique de concept élevé peut surclasser une citation publiée dans un périodique qui n'a pas la même reconnaissance conceptuelle. 
Figure 2 - Resultats de la recherche bibliométrique dans la base de données SCOPUS

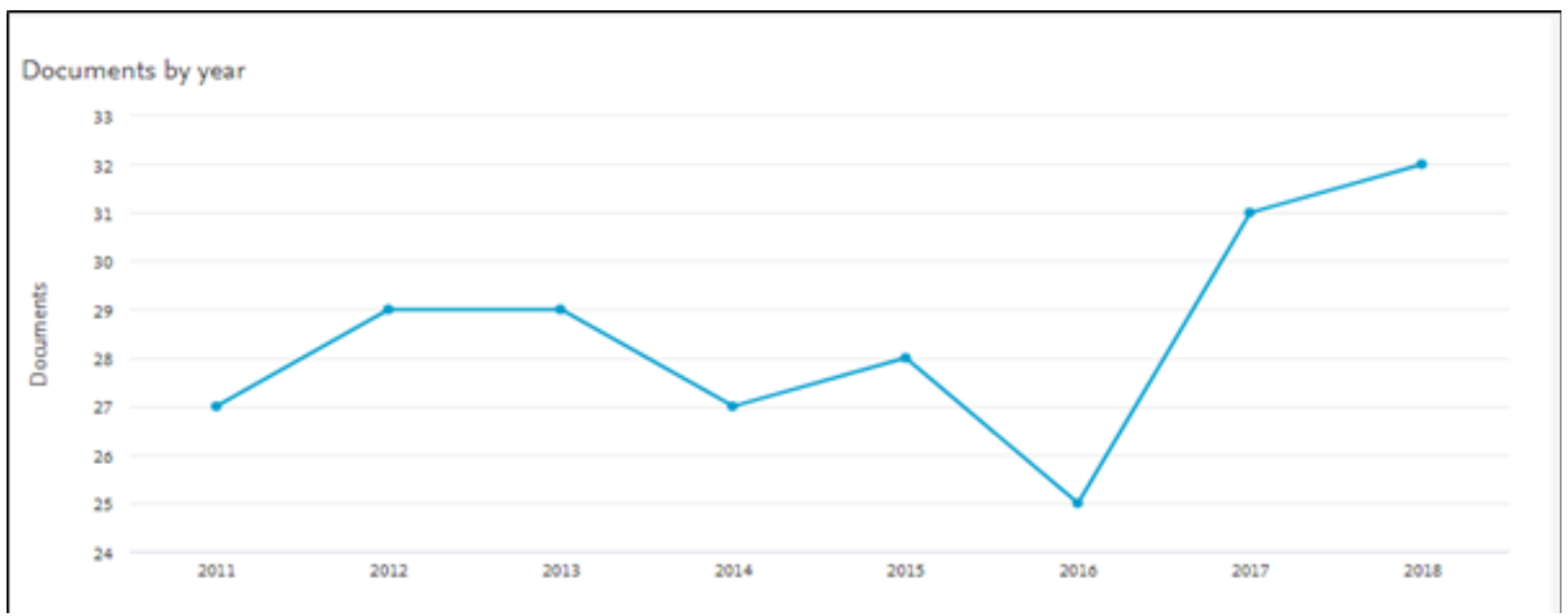

Source: Scopus (2018)

Malgré ses limites, la bibliométrie adapte une mesure fondamentalement objective de production scientifique (OKUBO, 1997). Dans les analyses bibliométriques s'utilise seulement la communication formelle entre les scientifiques: Articles, livres, brevets et documents, et les communications ne sont pas considérées comme informelles (littérature grise), tels que des présentations orales, des rapports, des annales de conférences et toute communication électronique non scientifique (YEN-TSANG; DULTRADE-LIMA; PRETTO, 2013).

En fonction de la loi de Zipf, qui mesure la fréquence des mots, le graphique introduit 228 documents résultant d'une recherche des mots-clés à propos de l'innovation radicale et l'innovation incrémentale, pour les années 2011 à 2018. On peut noter à partir de l'année 2016 une forte hausse dans les publications, confirmant un intérêt accru pour le sujet. Selon la loi de Lotka, qui étudie la productivité des auteurs, l'auteur le plus cité est Paavo Ritala, avec cinq articles. Cet auteur a plus de 80 documents produits dans la base de données et a été cité dans 881 autres articles, ce qui lui attribue un facteur $\mathrm{H}$ de 16. La loi de Bradford enquête sur la production présentée par les journaux scientifiques indexés dans les base de données. Le journal le plus cité est le Journal of Product Innovation Management avec 15 articles relatifs aux mots-clés recherchés et avec un indice SJR de 3,043. 


\section{MÉTHODE DE RECHERCHE}

La méthode de recherche utilisée propose une approche qualitative, de nature appliquée, en vue d'une recherche bibliométrique. Quant aux objectifs de la recherche, ils se classifient comme exploratoire et descriptif, qui appliquent des méthodes d'observation, d'enregistrement, de classification, d'analyse et d'interprétation des résultats, en utilisant la base de données Scopus. L'objectif de la recherche exploratoire se réalise sur un objet de recherche, lorsqu'il y a absence d'étude ou pas suffisamment d'études en ce qui concerne l'objet de recherche en question. En effet, l'objectif de ce type d'étude est de chercher des normes, des idées ou des hypothèses, pourtant, ne vise pas de les tester ou de les confirmer (COLLIS; HUSSEY, 2005).

Quant aux procédures la recherche peut être documentaire, c'est-à-dire, elle consiste en la collecte, la classification, la sélection diffuse et l'utilisation de toute espèce d'informations notamment dans la forme de textes et d'images (FACHIN, 2001). L'échatillon de la recherche a été obtenu par une sélection d'articles en accédant à la base de données Scopus, une base de données internationale qui offre des informations scientifiques à la communauté universitaire. Ainsi, la base de données Scopus est l'une des plus grande source référentielle de la littérature technique et scientifique, qui permet d'obtenir une vision élargie sur tout ce qui est publié scientifiquement sur un sujet, disponibilisant ainsi à une équipe de chercheurs une quantité d'informations suffisantes pour créer des projets, depuis la recheche fondamentale et appliquée, jusqu'à l'innovation technologique (ELSEVIER, 2018).

La recherche de publications repertoriées dans la base de données Scopus couvre la période de 2011 à 2019. L'univers de recherche comprend les publications rassemblées dans la base de données des sciences sociales et humaines, totalisant 218 articles. Les mots-clés utilisés ont été par titre, résumé et mots-clés, soit: radical innovation et incremental innovation. Après lecture des résumés, les articles en ordre de la plus haute citation ont été sélectionnés pour une lecture et discussion.

\section{ANALYSE DES RÉSULTATS}

Après l'analyse des articles résultants de la bibliométrie, on peut observer les différentes perspectives d'approche en ce qui concerneles énoncés sur les innovation radicale (IR) et incrémentale (II), tout comme l'ambidestrie des organisations dans la réalisation de l'exploration des produits existants pour permettre l'innovation incrémentale et explorer de nouvelles opportunités afin de promouvoir l'innovation radicale (ANDRIOPOULOS; LEWIS, 2009). La conceptualisation basée sur des modèles d'affaires et le rôle pour établir un lien direct entre le changement du modèle d'affaire et le degré d'innovation incrémentale versus 
radicale, distingue et spécifie les différents types de changement de modèle d'affaire (CAVALCANTE; KESTING; ULHOI, 2011).

II y a un manque d'études sur les innovations organisationnelles, les différences entre les drivers pour les innovations radicales ou incrémentales peuvent être d'étape et non d'espèce (DROEGE; HILDEBRAND; FORCADA, 2009). La portée du marché est un facteur important dans les produit et processus d'innovation. La mise en oeuvre des changements corporatifs a un impact positif sur l'innovation deproduit radical en mettant en œuvre de nouvelles structures organisationnelles et, a un effet positif sur l'innovation incrémentale (RADAS; BOŽIĆ, 2009).

Ainsi, lorsque des développements incrémentaux sont entrepris dans le cadre de la compétitioncoopération, les entreprises doivent non seulement chercher l'échange de connaissances pour créer de la valeur, mais aussi se rappeler à protéger le noyau de connaissances spécifiques dans l'entreprise. Afin de se maintenir compétitive, une entreprise doit constamment être à la recherche d'innovations afin de circonscrire les connaissances déjà existantes. Ces nouvelles innovations sont gératrices d'opportunités qui surgissent sur le marché (RITALA; HURMELINNA-LAUKKANEN, 2013)

La compétition-coopération des entreprises avec succès multiplie les opportunités d'innovation radicale, tandis que la compétition-coopération dyadique est la plus adéquate pour l'innovation incrémentale. Les differents objectifs d'innovation conduisent à des différents niveaux de création des valeurs, tensions, ainsi que d'appropriation entre concurrents-coopérateurs. Les différents niveaux du capital social lié au choix des partenaires sont nécessaires, pour que les concurrents aient du succès dans leur recherche d'innovations radicale ou incrémentale (YAMI; NEMEH, 2014).

Basé sur la théorie de l'économie des coûts de transaction et le rôle des fournisseurs dans l'intelligence du marché, ce processus diffère en ce qui concerne la nature du projet d'innovation (incrémentale versus radicale). La participation à de telles activités aura un plus grand impact sur la performance du produit remarqué dans l'innovation radicale que dans l'innovation incrémentale (SONG; THIEME, 2009).

La connaissance de la technologie a un effet sur la propension des utilisateurs de contribuer avec des idées nouvelles, qu'elles soient incrémentales ou radicales. Ces idées, générées par des utilisateurs, tendent à être incrémentales tandis que les idées des utilisateurs pionniers sont plutôt radicales. L'engagement des utilisateurs pionniers peut vraiment être une motivation pour la révision de la stratégie des affaires de l'entreprise (MAGNUSSON, 2009).

Si une entreprise utilise l'orientation pour le client, l'entreprise a une plus grande probabilité d'adopter l'innovation incrémentale, tandis que, si une entreprise utilise l'orientation compétitive, l'entreprise a la plus grande probabilité d'adopter l'innovation radicale. Enfin, les innovations radicale et incrémentale 
mènent à une plus grande performance du marché et l'amélioration de la performance financière (CHENG; KRUMWIEDE, 2010). Les entreprises d'aujourd'hui ne peuvent plus seulement se concentrer sur les besoins des clients, ou seulement sur l'anticipation des besoins de demain. Elles doivent être excellentes dans les deux contextes et cela nécessite la supervision de deux actes en équilibre: d'une part exceller dans l'utilisation et l'exploitation de ses capacités, et d'autre part, être excellent à la fois dans la gestion des innovations incrémentale et radicale (CORSO; MARTINI; PELLEGRINI, 2009).

L'effet de la capacité d'innovation incrémentale dans la performance du nouveau produit est positif sous une structure formelle, tandis que le même effet est négatif dans une structure informelle. Ainsi, les innovations radicale et incrémentale sont vues sous la vision de capacité de développement des innovations (MENGUC; AUH, 2010).

La vision de l'entreprise basée sur les connaissances, les alliances et les configurations alternatives de parenté technologique en recherche et développement inter-entreprises (R \& D) influencent des types spécifiques d'innovation des produits, où les alliances complémentaires contribuent au développement tant de l'innovation radicale que de l'innovation incrémentale (QUINTANA-GARCÍA; BENAVIDES-VELASCO, 2008). La gestion intellectuelle, la génération d'innovations radicales et incrémentales sont considérées comme des conséquences de gestion de la propriété intellectuelle (ROY; SIVAKUMAR, 2011).

Le système des pratiques de gestion organisationnelle permet une meilleure réalisation d'innovations radicales, tandis que le système des pratiques de gestion de niveau d'équipe atteint des meilleures innovations incrémentales et en utilisant les deux systèmes aide dans les innovations incrémentales, mais pas dans les innovations radicales. Par conséquent, les entreprises doivent gérer leurs employés différemment selon le type d'innovation qu'elles souhaitent atteindre (UN, 2010). Elles doivent se baser sur les théories de flux de la connaissance inter-organisationnelle et d'apprentissage organisationnel. La qualité et la fréquence de la recherche ouverte sont positivement liées à la performance de l'innovation incrémentale de l'entreprise. La complexité et la profondeur de la recherche sont positivement liées à la performance de l'innovation radicale (CHIANG; HUNG, 2010).

La dichotomie que présentent les innovations radicale et incrémentale se rapporte au degré de variation associé à l'innovation où l'impact organisationnel est perçu à partir du risque et du noyau des compétences organisationnelles existantes (CHETTY; STANGL, 2010). Le degré d'innovation peut varier entre les processus de développement des nouveaux produits incrémentaux et radicaux, et en relation avec l'impact des différentes structures fonctionnelles et interfonctionnelles, pour différents types de développement de nouveaux produits (DNP), processus (incrémental versus radical) et, en fonction de différents niveaux de performance de l'entreprise (DE VISSER et al., 2010). 
L'intégration fonctionnelle est un phénomène complexe qui ne permet pas d'apporter une conclusion générale en termes d'impact de performance. Ainsi, l'impact de l'intégration de la recherche et du développement, le marketing et les fonctions de production sur l'efficacité et l'efficience des projets de DNP se présentent dans un aspect de processus, de natures radicale ou incrémentale (BRETTEL et al., 2011). Les entreprises conservatrices entendent que les approches radicales de DNP, comme la nouveauté du produit et l'incertitude dans l'innovation de nouveaux produits/production peuvent supprimer et ne pas augmenter les barrières à la fabrication incrémentale et/ou radicale (SMITH, 2011).

Dans un contexte d'incertitude, les innovations radicale et incrémentale sont abordées dans la relation entre l'utilisation de l'ingénierie simultanée et de succès dans le développement de nouveaux produits, par l'intégration interfonctionnelle et le travail en équipe. En ce qui concerne les innovations incrémentales, elles affectent positivement la performance du DNP, en termes du temps de développement. Dans le cas des innovations radicales, elles affectent positivement la performance du DNP, en termes de coûts de développement (VALLE; VÁZQUEZ-BUSTELO, 2009). L'apprentissage de nouveaux attributs (radicaux) et I'ajout de caractéristiques aux attributs existants (incrémentaux) représentent deux étapes et différents degré de gestion du processus d'innovation, où les innovations radicales et incrémentales sont en corrélation (MARTINEZ-ROS; ORFILA-SINTES, 2009).

Les innovations radicales sont le fruit de la recherche fondamentale et d'un quota significativement plus important de recherche scientifique dans les organisations (STERNITZKE, 2010). Différentes approches pour le contrôle de gestion sont employées à différentes phases du processus d'innovation radicale et dans des projets d'innovation, montrant différents degrés d'innovation (CHIESA; FRATTINI; LAMBERTI; NOCI, 2009; CHANG; GONG; SHUM, 2011).

Par rapport à l'innovation ouverte, différentes activités d'innovation ouverte sont bénéfiques, gérant différents résultats d'innovation, tel que la « technologie de la sous-traitance «, qui est liée à la performance de l'innovation radicale et, la « technologie de mesure «, qui est liée à la performance de I'innovation incrémentale (FARIDA; WESTERBERG; FRISHAMMAR, 2012).

L'ensemble des pratiques de gestion de qualité à travers de la gestion de processus a une relation positive sur les cinq types d'innovation: radicale de produits, radicale de processus, incrémentale de produit, incrémentale de processus et innovation administrative. Ainsi, les innovations radicale et incrémentale peuvent être répertoriées comme des types d'innovation (WAGNER; MORTON DAINTY; BURNS, 2011; KIM; KUMAR; KUMAR, 2012). Aucun des ensembles de pratiques de gestion de la qualité totale (GQT) affecte directement l'innovation radicale, alors qu'ils ont une relation significative et positive avec l'innovation incrémentale. Le changement culturel contribue comme un facteur de la médiation, 
où toutes les relations sont significatives et où le pouvoir de changement culturel lie l'adversité des pratiques de GQT aux innovations incrémentale et radicale (MORENO-LUZON; GIL-MARQUES; VALLSPASOLA, 2013).

La créativité organisationelle naît à partir d'un amalgame d'innovations radicale et incrémentale, à travers une différenciation entre les routines mises en places pour la génération de performances créatives. La créativité peut avoir deux profils distincts: la créativité radicale, où la disposition pour assumer des risques, la disposition de ressources pour mettre en place une culture de créativité et l'engagement de carrière, et la créativité incrémentale qui implique que la présence de collaborateurs créatifs et l'identification organisationnelle sont associées à la créativité (MADJAR; GREENBERG; CHEN, 2011).

Le capital humain, le capital relationnel, la performance innovatrice de réseau d'innovations radical et incrémental par rapport à la diversité d'un réseau de connaissances, et le niveau de scolarité constituent des éléments importants pour améliorer toutes les composantes de la performance innovatrice (WINCENT; ANOKHIN; ÖRTQVIST, 2010). Les innovations radicale et incrémentale sont vues comme étant des générateurs de technologie industrielle afin de développer des sources de transformation organisationnelle (ZAPATA; NIEUWENHUIS, 2010).

En ce sens, l'innovation radicale se réfère à des innovations qui sont nouvelles pour l'entreprise, le marché et l'industrie, qui incorporent une technologie différente et nouvelle et, qui fournissent le plus grand bénéfice aux clients en ce qui concerne les produits actuels du secteur. En revanche, les innovations incrémentales se réfèrent aux améliorations de l'offre de produits existants d'une entreprise qui satisfait au mieux les besoins de ses clients actuels et potentiels, c'est-à-dire, les innovations incrémentales sont comme des adaptations, des perfectionnements, des améliorations ou des extensions de gamme, incorporant des nouvelles ressources qui offrent des avantages additionnels (VARADARAJAN, 2009).

La littérature sur les variétés du capitalisme et des systèmes nationaux d'innovation proposent des arguments sur la façon dont les entreprises requièrent différents types de qualifications professionnelles dans la poursuite des stratégies d'innovation de produit. Elle introduit des combinaisons spécifiques de connaissances scientifiques compatible avec les habilités des salariés comme incitatifs à l'innovation radicale de produit, à l'innovation incrémentale et à des stratégies d'imitation de produit (HERRMANN; PEINE, 2011).

Quatre modes d'innovation diffèrent dans leur portée de nouveauté (génération d'innovations et d'adoption) et dans leur degré de changement (radical et incrémental). L'orientation pour le marché influence positivement le nombre des générations et l'adoption d'innovations incrémentales. Les environnements hautement complexes améliorent l'introduction d'innovations radicale et incrémentale 
générées à l'interne et préjudicient l'adoption d'innovations incrémentales pour les entreprises orientées vers le marché (PÉREZ-LUNO; CAMBRA, 2013). D'une part, l'accumulation de connaissances non codifiées par des sources internes améliore de façon significative la capacité de l'entreprise à développer des innovations radicales, et d'autre part, la codification de la connaissance accélère le développement des innovations incrémentales (GARCIA-MUINA; PELECHANO-BARAHONA; NAVAS-LÓPEZ, 2009).

Les processus d'innovation pour le développement durable ont reçu une attention croissante dans la littérature académique, où des entreprises durables qui intégre leurs processus d'orientation et d'innovation montrent la création de valeurs dans le développement de nouveaux produits sur le marché (BOS-BROUWERS, 2010).

Pour récolter les avantages de l'innovation stratégique pour le développement, la formation d'équipes autonomes devient plus efficaces dans une perspective de gestion de projets à nouveauté technologique élevée ou dans une perspective d'innovation radicale. Des équipes plus dirigées génèrent de meilleurs résultats dans l'exécution du développement de l'innovation incrémentale (PATANAKUL; CHEN; LYNN, 2012; SZEKELY; STREBEL, 2013). L'innovation stratégique pour le développement organisationnel axé vers le marché futur a une relation directe avec le succès de l'innovation radicale. Ainsi, les processus utilisés dans les phases initiales de DNP pour les différents types de projets d'innovation sont des innovations incrémentales, des innovations technologiques, des innovations du marché et des innovations radicales (LINDGREN; O'CONNOR, 2011). Ce niveau de complexité démontre qu'il est important de développer la compréhension de la gestion des porte-feuilles d'innovation en couvrant une gamme de types et degrés d'innovation (BAREGHEH; ROWLEY; SAMBROOK; DAVIES, 2012).

Pour récolter les avantages de l'innovation systémique pour le développement, la trajectoire de l'innovation réussie d'un acteur de la chaîne de valeur démontre comment une collaboration coordonnée peut aider à générer une innovation incrémentale (technologique) pour développer un système modulaire et démontre l'importance des fournisseurs comme des acteurs dans le développement de l'innovation. Par conséquent, pour introduire avec succès l'innovation, les acteurs ont besoin d'unir leurs forces avec les autres organisations afin de répondre aux défis de l'innovation systémique (MLECNIK, 2013).

En abordant l'innovation tout au long du cycle de vie de l'entreprise, les décisions de celle-ci changent tout au long du temps et dans les différents stades de croissance. Les stratégies d'innovation sont classifiées dans les termes du type d'innovation (produit ou processus) et fonction de son degré de nouveauté (incrémental ou radical). Du croisement de ces types de décision, quatre stratégies d'innovation émergent: le développement de produits, l'apprentissage au travers de l'expérience, la découverte et la restructuration (EIRIZ; FARIA; BARBOSA, 2013). 
Finalement, une approche croisée dans l'analyse des résultats permet d'identifier que les innovations radicale et incrémentale sont traitées dans la littérature comme une classification de l'innovation basée sur la dimension des produits/services et en fonction de secteurs économiques distincts.

\section{CONCLUSION}

La répétition théorique présente l'analyse des typologies d'innovation sous la perspective du principe du chaos, à partir de la littérature qu'aborde les innovations radicale et incrémentale. La représentation de Brown e Eisenhardt (2004) pour analyser des articles qui traitent de I'IR et II. On peut conclure qu'il y a des similitudes et des divergences entre la classification de l'innovation, c'est-à-dire, de par la manière dont sont attribuées les innovations radicale et incrémentale, ses dimensions, ses degrés, ses typologies, ses technologies, ses projets, sa gestion, et, selon que ces concepts sont traités dans les segments industriels comme résultat d'idées et de processus d'affaires. Ainsi, à partir de la théorie du chaos, il est possible de prétendre qu'il existe deux façons d'interpréter les approches récentes des IR et II.

La première forme d'interpretation est négative dans la mesure où, même en partant des auteurs qui conceptualisent les IR et II, il se trouve qu'il existe encore une différence de sens et de cadrage pour les deux approches. Cela génère une certaine confusion quant à la signification réelle de ces deux classifications d'innovation. Cependant, la seconde forme d'interprétation est positive, car l'innovation désigne en elle-même un changement et une constante évolution. Ainsi, la diversité de classifications et d'approches théoriques contribue à l'enrichissement théorique face au progrès des IR et II dans différents secteurs de recherche en économie.

Bien que les dimensions ont été separées et définies, les considérations de chaque catégorie s'influencent entre elles, formant un aperçu d'ensemble de l'analyse du système d'innovation sous la vision de la théorie du chaos. La bureaucratie représente une réalité d'affaire qui met en évidence des stratégies qui exigent, interdisent ou limitent l'innovation. Ce fait souligne la nécessité de maintenir les systèmes d'innovation flexibles.

Une limitation de la recherche se trouve dans la période délimitée des articles abordés dans l'analyse, parce qu'elle a été réalisée dans un seul moteur de recherche d'articles scientifiques. Finalement, les autres applications de la théorie du chaos doivent encore être explorées, où la promotion de recherches futures devient essentielle pour l'avancement de la relation théorique entre les innovations radicale et incrémentale. 


\section{RÉFÉRENCES}

ANDRIOPOULOS, C.; LEWIS, M. W. Exploitation-Exploration Tensions and Organizational Ambidexterity: Managing Paradoxes of Innovation. Organization Science, v. 20, n. 4, p. 696-717, 2009.

ARAÚJO, C. A. Bibliometria: evolução histórica e questões atuais. Em Questão, Porto Alegre, v. 12, n. 1, p. 11-32, jan./jun. 2006. Disponivel em: http://revistas.univerciencia.org/index.php/revistaemquestao/ article/viewFile/3707/3495>. Consulté le: 10 jul. 2014.

ATUAHENE-GIMA, K.; SLATER, S. F.; OLSON, E. M. The contingent value of responsive and proactive market orientations for new product program performance. Journal of Product Innovation Management, v. 22, n. 6, p. 464-482, 2005.

BAREGHEH, A.; ROWLEY, J.; SAMBROOK, S. Innovation in food sector SMEs. Journal of Small Business and Enterprise Development, v. 19, n. 2, p. 300-321, 2012.

BELDERBOS, R.; CARREE, M.; LOKSHIN, B. Cooperative R\&D and firm performance. Research Policy, v. 33, n. 10, p. 1477-1492, 2004.

BOS-BROUWERS, H. E. J. Corporate Sustainability and Innovation in SMEs: Evidence of Themes and Activities in Practice. Business Strategy and the Environment. n. 19, p. 417-435, 2010.

BOUNCKEN, R. B.; FREDRICH, V.; RITALA, P. ; KRAUS, S. Coopetition in New Product Development Alliances: Advantages and Tensions for Incremental and Radical Innovation. British Journal of Management, v. 29, p. 391-410, 2018.

BRETTEL, M.; HEINEMANN, F.; ENGELEN, A.; NEUBAUER, S. Cross-functional integration of R\&D, marketing, and manufacturing in radical and incremental product innovations and its effects on project effectiveness and efficiency. Journal of Product Innovation Management, v. 28, n. 2, p. 251-269, 2011.

BROWN, S. L.; EISENHARDT, K. M. Estratégia competitiva no limiar do caos: uma visão dinâmica para as transformações corporativas. São Paulo: Cultrix, 2004.

CAVALCANTE, S.; KESTING, P. ; ULHOI, J. Business model dynamics and innovation: (re) establishing the missing linkages. Management Decision, v. 49, n. 8, p. 1327-1342, 2011.

CHANG, S.; GONG, Y.; SHUM, C. Promoting innovation in hospitality companies through human resource management practices. International Journal of Hospitality Management, v. 30, n. 4, p. 812-818, 
2011.

CHENG, C. C.; KRUMWIEDE, D. The effects of market orientation and service innovation on service industry performance: An empirical study. Operations Management Research, v. 3, n. 3-4, p. 161-171, 2010.

CHETTY, S. K.; STANGL, K. M. Internationalization and Innovation in a network relationship context. European Journal of Marketing, v. 44, n. 11-12, p. 1725-1743, 2010.

CHIANG, Y. H.; HUNG, K. P. Exploring open search strategies and perceived innovation performance from the perspective of inter-organizational knowledge flows. R \& D Management, v. 40, n. 3, p. 292-299, 2010.

CHIESA, V.; FRATTINI, F.; LAMBERTI, L.; NOCI, G. Exploring management control in radical innovation projects. European Journal of Innovation Management, v. 12, n. 4, p. 416-443, 2009.

CHRISTENSEN, C. M. The rules of innovation. Technology Review, v. 105, n. 5, p. 32-38, 2002.

, C.M. The Innovator's Dilemma: when New Technologies Cause Great Firms to Fail. 1. ed. New York: Collins Business Essentials, 2006.

Coccia, M. Driving forces of technological change in medicine: Radical innovations induced by side effects and their impact on society and healthcare. Technology in Society, v. 34, n. 4, p. 271-283, 2012.

COLLIS, J.; HUSSEY, R. Pesquisa em administração: um guia para alunos de graduação e pós-graduação. 2. ed. Porto Alegre: Bookman, 2005.

CORSO, M.; MARTINI, A.; PELLEGRINI, L. Innovation at the intersection between exploration, exploitation and discontinuity. Int. J. of Learning and Intellectual Capital, v. 6, n. 4, p. 324-340, 2009.

COUTINHO, L.; FERRAZ, J. C. Estudo da competitividade da indústria brasileira. 4. ed. Campinas: Papirus, 1995.

DE VISSER, M.; FAEMS, D.; ANDRIES, P. ; LOOY, B. V. Technology alliance port-folios and financial performance: value-enchancing and cost-increasing effects of open innovation. Journal of Product Innovation Management, v. 27, n. 6, p. 785-796, 2010.

DEWAR, R. D.; DUTTON, J. E. The adoption of radical and incremental innovations: An empirical analysis. 
Manage. Sci., v. 32, n. 11, p. 1422-1433, 1986.

DOSI, G. Technological paradigms and technological trajectories: a suggested Interpretation of the determinants and directions of technical change. Res. Policy., v. 11, n. 3, p. 147-162, 1982.

DROEGE, H.; HILDEBRAND, D.; FORCADA, M. A. H. Innovation in Services: Present Findings, and Future Pathways. Journal of Service Management, v. 20, n. 2, p. 131-155, 2009.

EIRIZ, V.; FARIA, A.; BARBOSA, N. Firm growth and innovation: Towards a typology of innovation strategy. Innovation: Management, Policy and Practice, v. 15, n. 1. p. 97-111, 2013.

ELSEVIER. Scopus. Disponível em: <http://www.americalatina.elsevier.com/corporate/scopus.php>. Consulté le: 12 août. 2018.

FACHIN, O. Fundamentos de metodologia. 3. ed. São Paulo: Atlas, 2001.

FARIDA, V.; WESTERBERG, M.; FRISHAMMAR, J. Inbound open innovation activities in high tech SMEs: the impact on innovation performance. Journal of Small Business Management, v. 50, n. 2, p. 283-309, 2012

FREEMAN, C. The National System of Innovation in historical perspective. Cambridge Journal of Economics, v. 19, n. 1, p. 5-24, 1995.

GARCIA, R.; CALANTONE, R. A critical look at technological innovation typology and innovativeness terminology: a literature review. J. Prod. Innov. Manage., v. 19, n. 2, p. 110-132, 2002.

GARCÍA-MUIÑA, F. E.; PELECHANO-BARAHONA, E.; NAVAS-LÓPEZ, J. E. Making the development of technological innovations more efficient: An exploratory analysis in the biotechnology sector. The Journal of High Technology Management Research, v. 20, n. 2, p. 131-144, 2009.

HENDERSON, R. M.; CLARK, K. B. Architectural innovation: the reconfiguration of existing systems and the failure of established firms. Adm. Sci., v. 35, n. 1, p. 9-30, 1990.

HERRMANN, A. M.; PEINE, A. When "national innovation system" meet "varieties of capitalism" arguments on labour qualifications: On the skill types and scientific knowledge needed for radical and incremental product innovations. Research Policy, v. 40, n. 5, p. 687-701, 2011.

KIM, D.Y.; KUMAR, V.; KUMAR, U. Relationship between quality management practices and innovation. 


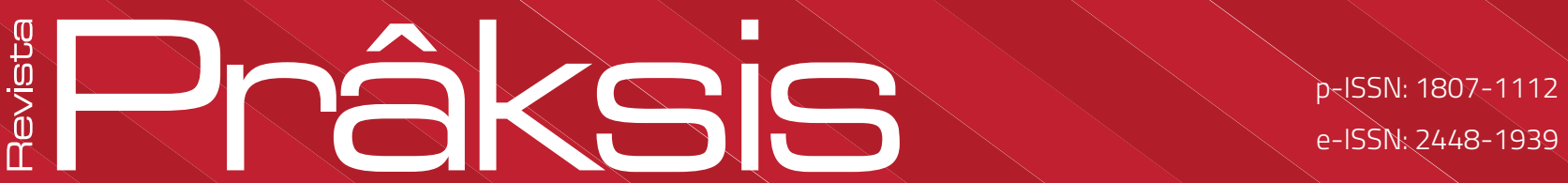

Journal of Operations Management, v. 30, n. 4, p. 295-315, 2012.

LEIFER, R.; MCDERMOTT, M. C.; O'CONNOR, G. C.; PETERS, L. S.; RICE, M. P. ; VERYZER, R. W. Radical Innovation. Boston: Harvard Business School Press, 2000.

LIMA, M. C.; TEIXEIRA; F. L. C. Inserção de um agente indutor da relação universidade-empresa em sistema de inovação fragmentado. Revista de Administração Contemporânea, v. 5, n. 2, p. 135-155, 2001.

LINDGREN, L. M.; O'CONNOR, G. C. The role of future-market focus in the early stages of NPD across varying levels of innovativeness. Journal of Product Innovation Management, v. 28, n. 5, p. 787-800, 2011.

MADJAR, N.; GREENBERG, E.; CHEN, Z. Factors for Radical Creativity, Incremental Creativity, and Routine, Non-creative Performance. J. Appl. Psychol., v. 96, n. 4, p. 730-743, 2011.

MAGNUSSON, P. R. Exploring the contributions of involving ordinary users in ideation of technology based services. Journal of Product Innovation Management, v. 26, n. 5, p. 578-593, 2009.

MARCH, J. G. Exploration and exploitation in organizational learning. Org. Sci., v. 2, n. 1, p. 71-87, 1991.

MARIETTO, M.; MEIRELES, M.; SANCHES, C.; SILVA, O. R. Teoria do Caos: Uma Contribuição para a Formação de Estratégias. In: XXX ENANPAD. Anais... Salvador, 2006.

MARTINEZ-ROS, E.; ORFILA-SINTE, S. F. Innovation activity in the hotel industry. Technovation, v. 29, n. 9, p. 632-641, 2009.

MCCARTHY, I. P. ; TSINOPOULOS, C.; ALLEN, P. ; ROSE-ANDERSSEN, C. R.New product development as a complex adaptive system of decisions. Journal of Product Innovation Management, v. 23, n. 5, p. 437456, 2006.

MEADOWS, D. Leverage Points: Places to Intervene in a System. Hartland, VT: Sustainability Institute, 1999.

MENGUC, B.; AUH, S. Development and return on execution of product innovation capabilities: The role of organizational structure. Industrial Marketing Management, v. 39, n. 5, p. 820-831, 2010.

MLECNIK, E. Opportunities for supplier-led systemic innovation in highly energy-efficient housing. 
Journal of Cleaner Production, v. 56, n. 1, p. 100-200, 2013.

MORENO-LUZON, M. D.; GIL-MARQUES, M.; VALLS-PASOLA, J. TQM, innovation and the role of cultural change. Industrial Management \& Data Systems, v. 113, n. 8, p. 1149-1168, 2013.

NELSON, R. R. National innovation systems: a comparative analysis. Oxford: Oxford University Press, 1993.

OCDE. "Organisation for Economic Co-operation and Development", Oslo Manual, <www.oecd.org/ dataoecd/35/61/2367580.pdf>, Consulté le 27 octobre 2010.

OKUBO, Y. Bibliometric indicators and analysis of research systems: methods and examples. Paris: OECD, 1997 (STI Working Papers, 1997/1).

O'REILLY, C. A.; TUCHMAN, M. L. The ambidextrous organization. Harvard Business Review, v. 82, n. 4, p. 74-81, 2004.

PATANAKUL, P. ; CHEN, J.; LYNN, G. S. Autonomous teams and new product development. Journal of Product Innovation Management, v. 29, n. 5, p. 734-750, 2012.

PÉREZ-LUNO, A.; CAMBRA, J. Listen to the market: do its complexity and signals make companies more innovative ? Technovation, v. 33, n. 6, p. 180-192, 2013.

POPADIUK, S.; CHOO, C. W. Innovation and knowledge creation: How are these concepts related ? International Journal of Information Management, v. 26, n. 4, p. 302-312, 2006.

QUINTANA, G. C.; BENAVIDES-VELASCO, C. A. Innovative competence, exploration and exploitation: The influence of technological diversification. Research Policy, v. 37, p. 492-507, 2008.

RADAS, S.; BOZIC, L. The antecedents of SME innovativeness in an emerging transition economy. Technovattion, v. 29, n. 6-7, p. 438-450, 2009.

RITALA, P. ; HURMELINA-LAUKKANEN, P. Incremental and Radical Innovation in Coopetition-The Role of Absorptive Capacity and Appropriability. Journal of Product Innovation Management, v. 30, p. 154-169, 2013. ROY, S.; SIVAKUMAR, K. Managing intellectual property in global outsourcing for innovation generation. The Journal of Product Innovation Management, v. 28, n. 1, p. 48-62, 2011.

ROY, S.; SIVAKUMAR, K.; WILKINSON, I. F. Innovation generation in supply chain relationships: a 
conceptual model and research propositions. Journal of the Academy of marketing Science, v. 32, n. 1, p. 61-79, 2004.

SMITH, A. D. Competitive approaches to new product development: A comparison of successful organizations in an unstable economic environment. Team Performance Management, v. 17, n. 3-4, p. 124-145, 2011.

SONG, M.; THIEME, J. The Role of Suppliers in Market Intelligence Gathering for Radical and Incremental Innovation. The Journal of Product Innovation Management, v. 26, n. 1, p. 43-57, 2009.

STACEY, R. D. A fronteira do caos. Venda Nova: Bertrand, 1995.

STAMM, B. Managing innovation, design \& creativity. London Business School: Wiley, 2003.

STERNITZKE, C. Knowledge sources, patent protection, and commercialization of pharmaceutical innovations. Research Policy, v. 39, n. 6, p. 810-821, 2010.

STREHL, L. O Fator de Impacto do ISI e a Avaliação da Produção Científica: aspectos conceituais e metodológicos. Ciência da Informação, v. 34, n. 1, p. 19-27, 2005.

SZEKELY, F.; STREBEL, H. Incremental, radical and game-changing: Strategic innovation for sustainability. Corporate Governance, v. 13, n. 5, p. 467- 481, 2013.

TUSHMAN, M.; NADLER, D. Organizing for innovation. California Manage. Rev., v. 28, n. 3, p. 74-92, 1986.

UN, C. A. An empirical multi-level analysis for achieving balance between incremental and radical innovations. Journal of Engineering and Technology Management - JETM, v. 27, n. 1-19, 2010.

VALLE, S.; VÁZQUEZ-BUSTELO, D. Concurrent engineering performance: Incremental versus radical innovation. International Journal of Production Economics, v. 119, n. 1, p. 136-148, 2009.

VARADARAJAN, R. Fortune at the bottom of the innovation pyramid: The strategic logic of incremental innovations. Business Horizons, v. 52, n. 1, p. 21-29, 2009.

YAMI, S.; NEMEH, A. Organizing coopetition for innovation: The case of wireless telecommunication sector in Europe. Industrial Marketing Management, v. 43, n. 2, p. 250-260, 2014. 
YEN-TSANG, C.; DULTRA-DE-LIMA, R. G.; PRETTO, K. Análise qualitativa das publicações nacionais e internacionais em etnografias em administração e estudos organizacionais. Administração: Ensino e Pesquisa, v. 14, n. 2, p. 211-247, 2013.

WAGNER, H. T.; MORTON, S. C.; DAINTY, A. R. J.; Burns, N. D. Path dependent constraints on innovation programs in production and operations management. International Journal of Production Research, $\mathrm{v}$. 49, n. 11, p. 3069-3085, 2011.

WINCENT, J.; ANOKHIN, S, ÖRTQVIST, D. Does network board capital matter ? A study of innovative performance in strategic SME networks. Journal of Business Research, v. 63, n. 3, p. 265-275, 2010.

ZAPATA, C.; NIEUWENHUIS, P. Exploring innovation in the automotive industry: new technologies for cleaner cars. Journal of Cleaner Production, v. 18, n. 1, p. 14-20, 2010. 